\title{
Téoros
}

Revue de recherche en tourisme

\section{La société touristique innu}

Du défi à la réalité

\section{Guylaine Gill}

Volume 17, numéro 2, été 1998

L'industrie touristique autochtone

URI : https://id.erudit.org/iderudit/1072272ar

DOI : https://doi.org/10.7202/1072272ar

Aller au sommaire du numéro

Éditeur(s)

Université du Québec à Montréal

ISSN

0712-8657 (imprimé)

1923-2705 (numérique)

Découvrir la revue

Citer cet article

Gill, G. (1998). La société touristique innu : du défi à la réalité. Téoros, 17(2),

28-30. https://doi.org/10.7202/1072272ar

Ce document est protégé par la loi sur le droit d'auteur. L'utilisation des services d'Érudit (y compris la reproduction) est assujettie à sa politique d'utilisation que vous pouvez consulter en ligne.

https://apropos.erudit.org/fr/usagers/politique-dutilisation/
Cet article est diffusé et préservé par Érudit.

Érudit est un consortium interuniversitaire sans but lucratif composé de l'Université de Montréal, l'Université Laval et l'Université du Québec à Montréal. Il a pour mission la promotion et la valorisation de la recherche. https://www.erudit.org/fr/ 


\section{Guylaine Gill}

Voilà plus de trente ans qu'on parle de prise en charge dans les milieux autochtones. Malgré les nombreuses embüches, les négociations territoriales ardues, les conditions sociales alarmantes, les déficiences en ressources humaines et matérielles, on constate un progrès significatif vers cette démarche d'autonomie si chère à nos peuples. Plusieurs secteurs sont maintenant gérés directement par les conseils de bande et de plus en plus d'organisations et d'entreprises voient le jour dans les différentes communautés du Québec. Des domaines comme l'éducation, les services sociaux, l'habitation, les communications, le développement économique, le transport aérien et les services de police ont graduellement été rapatriés. Bien que leur gestion puisse être critiquée, on doit reconnaître que des pas de géant ont été franchis puisqu' on assiste enfin à la naissance de programmes et de services modelés selon les réalités culturelles et les priorités sociales et économiques des Nations. C'est dans cette perspective que s'inscrivent les initiatives de la Société touristique Innu (STI) dans le domaine touristique, secteur encore jeune offrant des moyens privilégiés de développement.

Bien que l'idée de développer le tourisme en milieu autochtone remonte à quelques dizaines d'années, ce n'est qu'en 1991 qu'était fondée officiellement la Société touristique Innu, première organisation régionale autochtone québécoise avec mission de développer le tourisme à caractère culturel. Pour l'instigateur (le groupe Gamac), il s'agissait d'amorcer la deuxième phase d'un projet dont l'objectif était de créer des activités propices au développement social et économique des communautés. La première étape avait été l'acquisition de transporteurs aériens situés près des régions habitées par les Atikamekw et les Montagnais (Mauricie, le Lac Saint-Jean et Cồte Nord). Plusieurs conseils de bande, organismes et individus autochtones devenaient ainsi co-propriétaires de trois entreprises aériennes dont les populations autochtones locales étaient les principales utilisatrices. Du même coup, on se dotait d'un moyen de communication essentiel pour transporter les éventuelles clientèles touristiques vers les communautés et les territoires visés.

\section{UN PROJET COMMUNAUTAIRE}

Le fonctionnement de la nouvelle organisation fut défini en s'appuyant sur une étude exhaustive du potentiel, des infrastructures touristiques et de la création d'un réseau touristique en milieu atikamekw et montagnais réalisée par la firme Entraco, en étroite collaboration avec les deux paliers de gouvernement. On devait tout mettre en ceuvre pour développer et promouvoir efficacement le tourisme dans les régions concernées. Le défi était lancé.

Incorporée en vertu de la troisième loi sur les compagnies, l'entreprise, à but non lucratif, avait pour premier objectif de sensibiliser les entreprises autochtones et les conseils de bande au potentiel touristique des communautés, à l'envergure et à la nature de ses retombées sociales et économiques et à l'importance du regroupement.

Les particularités culturelles des autochtones, jumelées à la diversité des communautés, à leur relation privilégiée avec la nature et à leur environnement géographique, constituent des attraits recherchés par la clientèle internationale en quête d'aventure et d'exotisme. Il était donc important de faire valoir auprès des membres des communautés, cette "plus value * dont elles pouvaient bénéficier dans un contexte de fort potentiel de croissance du tourisme d'aventure. Il fallait également montrer qu'un développement touristique bien planifié pouvait constituer un atout socioéconomique important en offrant aux populations locales la possibilité de travailler dans un domaine qui mette en valeur leur culture, leur langue et leur mode de vie. Finalement, c'était une occasion privilégiée d'échanger sur les préoccupations des intervenants concernés et d'identifier les aspects sur lesquels il fallait mettre l'accent.

Dès la première assemblée générale officielle, à l'automne 1995, plusieurs mandats furent confiés à la STI. Les activités de l'organisation devaient porter principalement sur l'amélioration des

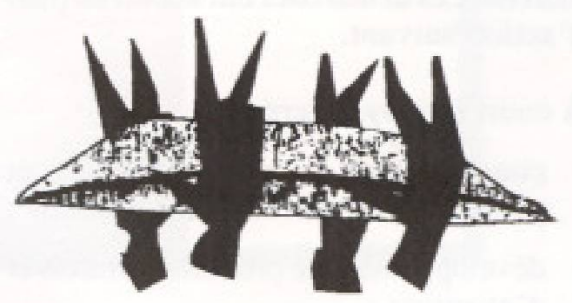




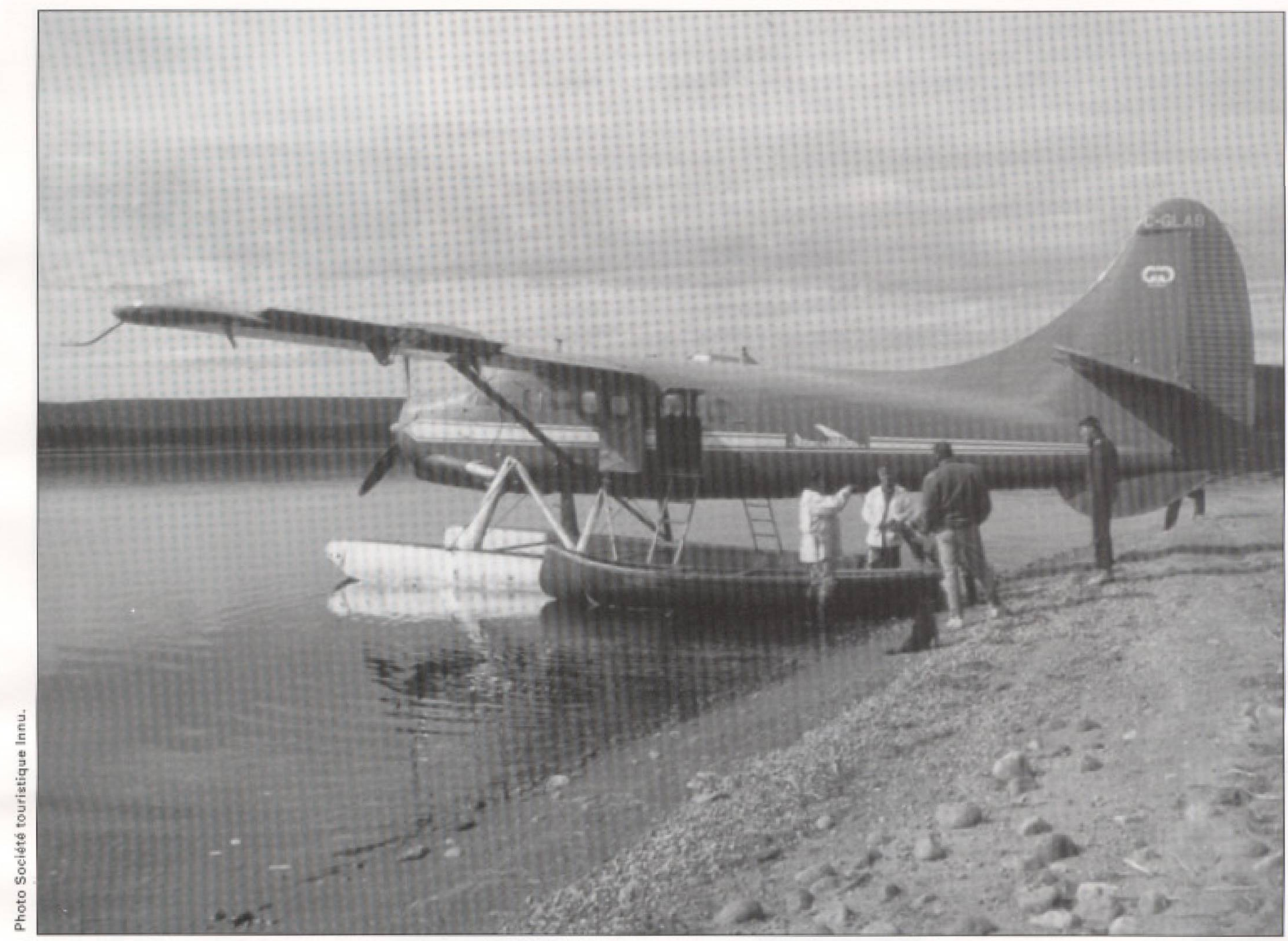

produits existants, l'assistance au développement de nouveaux produits et la mise en marché des produits prêts à être commercialisés.

\section{UN BÉMOL DANS LE DÉVELOPPEMENT}

La démarche de structuration de l'offre touristique et la commercialisation comportent plusieurs aspects et exigent une réflexion toute particulière si l'on veut améliorer les conditions sociales des communautés en mettant en valeur une richesse aussi précieuse que la culture. Conséquemment, et pour répondre à la principale préoccupation des populations autochtones concernant la sauvegarde de la culture, il était impératif pour les dirigeants de s'assurer que leurs actions seraient cohérentes et n' entraîneraient pas un développement chaotique susceptible de sombrer dans une quête lucrative dé- tachée de l'objectif premier. C'est donc avec un souci constant de contribuer effectivement à la pérennité de la richesse culturelle des premières nations que les activités de la STI ont été planifiées.

\section{PRINCIPALES RÉALISATIONS}

En moins de trois ans et malgré d'importantes contraintes (surtout financières), la STI jetait les bases d'une organisation touristique reconnue. Parmi ses nombreuses réalisations, notons l'élaboration et l'implantation officielle d'un cahier de normes de qualité et de sécurité destiné à garantir les produits auprès de la clientèle, la planification et la coordination de programmes de formation liés aux différents champs d'intervention touristiques et adaptés aux besoins du milieu, la mise sur pied d'un grossiste spécialisế en produits autochtones (Tours Innu), la conception de plusieurs circuits touristiques autoch- tones, le développement des outils promotionnels nécessaires à la commercialisation, la présentation des produits sur la scène internationale par des représentations particulières et la participation à plusieurs bourses et foires touristiques d'envergure (Atelier Canada, World Travel Market, ITB, Rendez-vous Canada, etc.). Au cours de cette période, la STI s'est également engagée activement dans l'industrie touristique québécoise, canadienne et internationale en participant à différents événements tels que les principaux forums, colloques et conférences.

\section{DES COLLABORATEURS INDISPENSABLES}

Pour la STI, la collaboration était essentielle. Ainsi, l'organisation a eu recours à l'appui de divers organismes et instances gouvernementales. Le gouvernement fédéral a contribué financièrement à plu- 
sieurs reprises. Le gouvernement provincial a également participé, notamment à l'étude de faisabilité initiale. Les communautés et les organisations autochtones ont quant à elles contribué d'une manière ponctuelle et très significative, particulièrement pour la réalisation des programmes de formation destinés aux membres de leurs populations.

En outre, plusieurs organisations et institutions ont travaillé avec la STI au cours des dernières années, dont le SACO, qui a souvent fourni les divers services professionnels de conseillers volontaires, Gestion Gamac PN (entreprise autochtone du secteur de l'aviation), qui a apporté une énorme contribution à toutes les phases de l'implantation de l'organisation et qui continue encore aujourd'hui d'apporter une aide ponctuelle, et Voyages InterNations (agence de voyage autochtone) avec laquelle le grossiste a été mis en place.

A I'automne 1997, la STI a conclu une entente de partenariat avec la Chaire de tourisme de l'Université du Québec à Montréal. Ainsi, la STI et ses membres ont pu entreprendre des activités telles que l'élaboration d'un plan de développement touristique pour la communauté de Manawan et d'un plan d'affaires pour le grossiste Tours Innu. Ce partenariat a également favorisé l'obtention de l'assistance professionnelle requise pour $\mathrm{l}^{\prime} \mathrm{im}$ plantation d'un centre d'interprétation historique pour la Société de développement de Betsiamites. Mentionnons finalement les efforts de Tourisme Québec et de la Commission canadienne du tourisme dans la promotion internationale des produits de la STI.

\section{ET APRÈS...}

La Société touristique Innu est maintenant à une étape déterminante de son développement, autant sur le plan commercial que de son action sur la scène touristique autochtone. D'abord, c'est au cours de cette année 1998 que se sont réalisées les premières ventes par Tours Innu. C'est également au cours de l'automne 1998 que des décisions importantes seront prises concernant la structure financière du grossiste.

Par ailleurs, la STI se trouve à un tournant quant à son engagement dans le développement de l'offre touristique autochtone.
Ainsi, lors de la conférence sur le tourisme autochtone tenue à Québec au printemps 1998 (dont on peut lire le rapport dans le présent numéro), il a été convenu que les autochtones du Québec ont avantage à unir leurs efforts pour développer leur industrie touristique. Plusieurs motifs ont alors éte évoqués dont certains sont incontournables, comme la disposition des gouvernements à ne financer qu' une seule organisation centrale. On notait aussi plusieurs éléments en faveur d'un regroupement : mise en commun des services et des expertises dans les domaines de la formation, de la commercialisation et de la représentation, standardisation de normes de qualité et de sécurité, etc. Il est également apparu avantageux de travailler ensemble au développement d'une offre diversifiée et complémentaire. Dans ses conclusions, l'assemblée donnait naissance à un cercle formé d'intervenants de différentes nations chargés de trouver un modèle de regroupement efficace et représentatif.

En conséquence, lors de la première réunion du cercle en juin dernier, les dirigeants de la Société touristique Innu, avec l'accord de leur assemblée générale, ont proposé la restructuration de la STI pour créer une organisation provinciale, permettant ainsi à tous les intervenants touristiques qui le souhaitent de bénéficier des ressources et des expertises déjà acquises à la STI. Un sous-comité est actuellement à l'ceuvre pour développer une proposition de restructuration. Il est fort possible donc que, dès cette année, une décision importante soit prise concernant I'avenir de la Société touristique Innu au profit d'une organisation desservant tous les intervenants touristiques autochtones du Québec intéressés à travailler en étroite collaboration à l'implantation d'une industrie mieux structuré.

Jusqu'à ce jour, les activités de la Société touristique Innu étaient principalement destinées au développement touristique sur les territoires des trois communautés atikamekw et des neuf communautés montagnaises. Les communautés atikamekw, dont la population totale est de 4500 personnes, forment un triangle ponctuant le nord-ouest de l'arrière-pays, de la Haute-Mauricie et de Lanaudière. II s'agit de Manawan, Wemotaci et Opiticiwan. Les communautés montagnaises, surtout situées en bordure du Saint-Laurent, comptent une population totale de

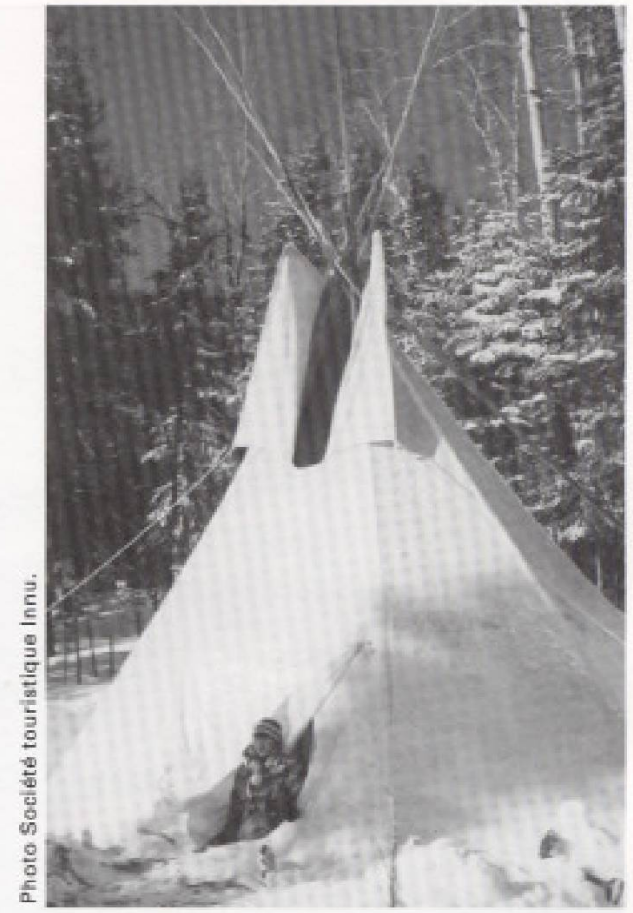

13000 personnes. Sur la Cồte Nord, on trouve les communautés de Pessamit, Essipit et Uashat Mak-Mani-Utenam et sur la Basse Côte Nord, Ekuantshit, Natashquan, Unamen Shipi et Pakua Shipi. Mashteuiatsh est située au Lac Saint-Jean et Matimekush est adjacente à Schefferville, au Nouveau-Québec.

Notons que le territoire couvert par les douze communautés recoupe les régions d'intervention de huit municipalités régionales de comté, de cinq associations touristiques régionales ainsi qu'une portion du Nouveau-Québec.

Guylaine Gill travaille depuis trois ans à titre de directrice générale de la Société touristique Innu. Elle s'est intéressée au dossier du tourisme en milieu autochtone des le debut, à titre de secrétaire executive et de chargée de projet pour Gestion Gamac PN. Elle a egalement collaboré étroitement à l'implantation de liaisons aériennes sur la Côte Nord. Originaire de Mashteuiatsh, Guylaine Gill s'est toujours intéressée au développement de structures el de systèmes adaptés aux besoins des conmunautes. En 1984, elle participait à l'établissement de la structure administrative du Régime des Bénéfices autochtone, organisme offrant des avantages sociaux chez les employeurs autochtones du Québec. Elle a dirige l'organisation pendant cinq ans et continue maintenant de collaborer à titre de vice-présidente.

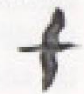

